\title{
FEATURE IMPORTANCE ANALYSIS OF SENTINEL-2 IMAGERY FOR LARGE-SCALE URBAN LOCAL CLIMATE ZONE CLASSIFICATION
}

\author{
Chunping Qiu ${ }^{1}$, Michael Schmitt ${ }^{1}$, Pedram Ghamisi ${ }^{2}$, Lichao Mou ${ }^{1}$, Xiao Xiang Zhu ${ }^{1,2}$ \\ ${ }^{1}$ Signal Processing in Earth Observation, Technical University of Munich (TUM), Munich, Germany \\ ${ }^{2}$ Remote Sensing Technology Institute (IMF), German Aerospace Center (DLR), Wessling, Germany
}

\begin{abstract}
This paper evaluates different spectral-spatial features that can be extracted from Sentinel-2 imagery regarding their relevance for discriminating different Local Climate Zone (LCZ) classes. The features include spectral reflectance, spectral indices, Morphological Profiles (MPs), as well as Global Urban Footprint (GUF), the Open Street Map layers buildings and land use, and their combinations. Using a residual convolutional neural network (ResNet), a systematic analysis of feature importance is performed with a manually generated dataset distributed in Europe. The results of this evaluation are meant to provide guidance about the choice of both spectral and spatial features for the task of LCZ classification on a global scale. The results show that GUF and OSM can contribute to the classification performance, and ResNet relies less on additional features with the highest accuracy provided by the reflectance only.
\end{abstract}

Index Terms - Local Climate Zones (LCZs), Sentinel2, Spectral features, Classification, Morphological Profiles (MPs), residual convolutional neural network (ResNet)

\section{INTRODUCTION}

Local Climate Zone (LCZ) mapping [1], originally developed for meta-data communication of observational Urban Heat Island (UHI) studies, also helps to classify weather stations and assess social inequalities. The $17 \mathrm{LCZ}$ classes are related to 3D surface structure (e.g. height and density of buildings and trees) as well as surface cover (e.g., vegetation or paved). Recently, researchers have started to use the LCZ scheme to classify the internal structure of urban areas, providing critical information for various applications, including urban climatology, infrastructure planning, disaster mitigation, etc. Besides, as an environmental factor, LCZs are expected to enable evidence-based strategies for planning healthy cities worldwide [2].

Supervised classification with remote sensing data provides a valuable tool for automatic LCZ mapping, as illustrated by the existing literatures [3, 4].However global LCZ mapping is still challenging due to the limited number of high quality ground truth, as well as a large intra-class variability of spectral signatures caused by the regional variations of vegetation and artificial materials and other variations in cultural and physical environmental factors [5].

For automatic global LCZ classification, the challenges are data availability and high generalization ability as well as transferability of the employed classification algorithm. Available datasets with high potential for this task include, but are not limited to, imagery (e.g., Landsat 8, Sentinel-1, and Sentinel-2), vector data (from OpenStreetMap, OSM) [3], and settlement layers (Global Urban Footprint, GUF) [6]. It is of great importance to know the specific potential of each of these datasets. However, few literatures exist in this regard. [4] investigated the feature importance for the ensemble of Canonical Correlation Forests (CCFs), showing that NDVI is the most important feature among the OSM layers (land use, building and water), spectral reflectance of Landsat 8 and spectral indices extracted using Landsat 8 imagery.

In this study, we focus on the globally available imagery provided by the Sentinel-2 mission [7], as well as GUF and OSM layers. Our work intends to investigate the usefulness and relative importance of the features fed to classifiers. Answers will be provided for these questions: What is the relative importance of different bands, index measures, and spatial features extracted from Sentinel-2, as well as the external auxiliary data (GUF and OSM layers)? How do GUF and OSM contribute to the classification performance? The answers will provide guidance for the right choice of proper features for large-scale LCZ classification. As a framework for our investigations, we use a Residual convolutional Neural Network (ResNet). For simplicity, we focus on the first ten LCZ classes, which are referred to as urban LCZ classes in this paper and we add two additional background classes, namely vegetation and water, to achieve land cover completeness.

\section{FRAMEWORK FOR FEATURE IMPORTANCE ANALYSIS}

In this section, a feature importance investigation has been designed with the purpose of: (i) evaluating the feature importance of the spectral reflectance, index measures and spatial information from Sentinel-2 imagery, as well as the exter- 
nal auxiliary GUF and two OSM layers with respect to urban LCZ classification; (ii) Assessing and comparing the applicability of GUF and OSM layers to urban LCZ classification. The investigated dataset is described first.

\subsection{Study Areas and LCZ Dataset}

Our study areas are cities located in the heart of Europe: Amsterdam, London, Berlin, Paris, Cologne, Munich and Milan. For all 7 cities, we have downloaded Sentinel-2 imagery from ESA's SciHub (https://scihub. copernicus.eu/). In addition, we are allowed to access DLR's Global Urban Footprint (GUF), a binary layer derived from TanDEM-X data, which indicates urban areas. Finally, we have downloaded the Open Street Map layers building and land-use from OpenStreetMap Data Extracts (http: / / download. geofabrik.de/) for each city. The LCZ ground truth labels available for selected neighborhoods in the 7 cities are taken from the LCZ42 dataset [8]. In these 7 cities, LCZ class 7 (lightweight low-rise), mostly indicating slums, does not exist. Details about the study areas and the dataset can be found in our previous work [9].

\subsection{Classification Framework}

For our investigations, we use a residual convolutional neural network (ResNet) as the classifier, as ResNet has been proven to be capable of offering better classification performance [10]. The exact architecture of the ResNet we train is shown in Fig. 2. Overall, it has three residual blocks, and each of them consists of three convolutional layers and a shortcut connection that by-passes two stacked convolutional layers by performing identity mapping, which are then added together with the output of stacked convolutions. We utilize convolutional layers with a very small receptive field of $3 \times 3$, and the number of feature maps increases towards deeper blocks, doubling after each block. Max-pooling is performed over $2 \times 2$ pixel windows with stride 2 . For training the network, we use the TensorFlow framework. We choose Nesterov Adam as optimization algorithm for our task, as it shows faster convergence than standard stochastic gradient descent with momentum. We fix parameters of Nesterov Adam as recommended: $\beta_{1}=0.9, \beta_{2}=0.999, \epsilon=1 \mathrm{e}-08$, and a schedule decay of 0.004 . We use a fairly small learning rate of 0.0002 .

Furthermore, we define 19 features as input to the classifier:

\section{- Spectral reflectance}

10 bands of Sentinel-2 imagery are used in this study: B2, B3, B4 and B8 with $10 \mathrm{~m}$ Ground Sampling Distance (GSD) and B5, B6, B7, B8a, B11 and B12 with $20 \mathrm{~m}$ GSD. The $20 \mathrm{~m}$ bands are up-sampled to $10 \mathrm{~m}$ GSD. The bands B1, B9 and B10 are not considered in this study because they contain mostly information about the atmosphere and thus bear little relevance to LCZ classification.

\section{- Indices}

The well-established indices Normalized Difference Vegetation Index (NDVI), Enhanced Vegetation Index (EVI), Modified Normalized Difference Water Index (MNDWI), Normalized Difference Built Index (NDBI) and Bare-Soil Index (BSI) are also considered [11], since they can provide indications about vegetation, water, buildings and soil, respectively [5].

\section{- Morphological profiles (MPs)}

Morphological profiles are constructed based on the repeated use of opening and closing by reconstruction with a Structuring Element (SE) of increasing size applied to images. It is able to attenuate irrelevant spatial details with respect to the shape and size of SE, decrease the intra-class variability, and preserve the geometrical characteristics of the input image in a simultaneous manner [12]. In this study, MPs with a circular $\mathrm{SE}$ and threshold values of 1,2, 3, 4 and 5 pixel are used to extract spatial information from the Sentinel-2 imagery.

\section{- Other auxiliary data}

As auxiliary data, both OSM and GUF are re-sampled to $10 \mathrm{~m} \mathrm{GSD}$.

These features for an exemplary patch are visualized in Fig. 1.

\subsection{Setups for feature importance analysis}

An analysis scheme is designed based on [3] which indicates that Normalized Difference Vegetation Index (NDVI) and Morphological profiles (MPs) are powerful features for LCZ mapping. Table 1 shows the feature combinations being investigated in this study. Comparing the results from configurations $R_{-} l$ to $R_{-} 4$, the relative importance of index measures, MPs, GUF and OSM can be interpreted. Besides, a comparative analysis of all classification results can provide a hint to the best configuration for large-scale LCZ mapping.

For all experiments, we rely on cross validation, i.e., each time samples from six cities are used for training while those from the seventh city are used for testing.

\section{EXPERIMENTAL RESULTS}

Classification results of different feature configurations (as described in Tab. 1) are compared and shown in Fig. 3.

Two exemplary LCZ maps from the configuration $R \_0$ and $R_{-} l$ in Tab. 1 , over the city of Munich, Germany and the Sentinel-2 imagery are shown in Fig. 4. 


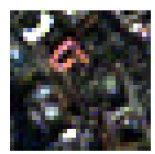

(a) RGB

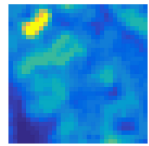

(k) O_R_1

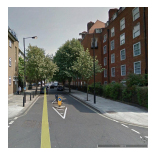

(b) GSV

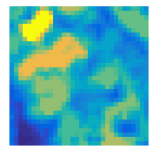

(1) O_R_2

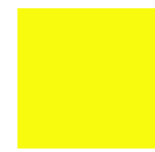

(c) GUF

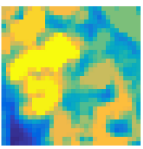

(m) O_R_3

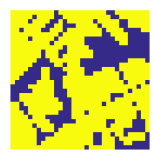

(d) OSM_b

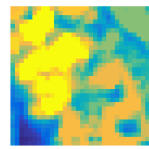

(n) O_R_4

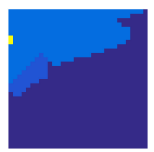

(e) OSM_lu

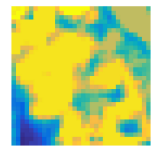

(o) O_R_5

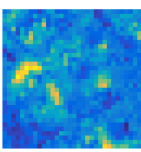

(f) EVI

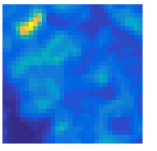

(p) $\mathrm{C} \_$R_1

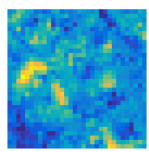

(g) NDVI

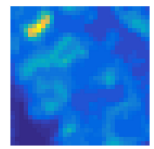

(q) $\mathrm{C} \_\mathrm{R} \_2$

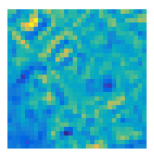

(h) NDBI

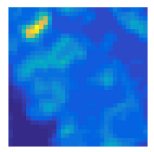

(r) $C_{-}$__3

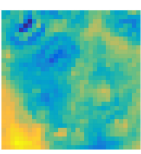

(i) MNDWI

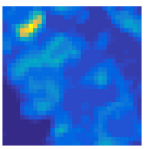

(s) C_R_4

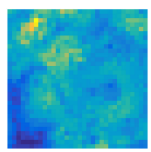

(j) BSI

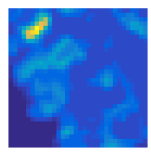

(t) C_R_5

Fig. 1: The features being investigated of an exemplary patch (LCZ 2, compact middle rise ) in London, England, as well as the Sentinel-2 RGB image (a) and Google street view (b). (c)-(e) are auxiliary data; (f)-(j) are index measures; (k)-(t) are MPs, where $O \_R \_k\left(k \in(1,5)\right.$ and $C_{-} R_{-} k(k \in(1,5)$ are closing and opening by reconstruction with a SE size of k pixel, respectively. The values of each feature are scaled to $(0,1)$.
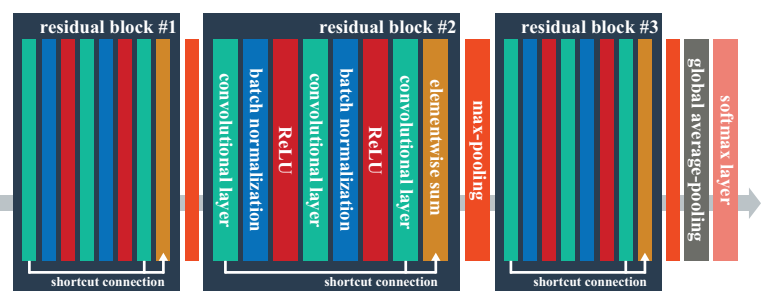

Fig. 2: The architecture of the used ResNet for LCZ classification.

Table 1: Comparative experiment ID and the corresponding selected features.

\begin{tabular}{ll}
\hline input feature & ID \\
\hline Spectral reflectance & $R \_0$ \\
Spectral reflectance, Indices & $R_{-} 1$ \\
Spectral reflectance, MPs & $R \_2$ \\
Spectral reflectance, GUF & $R_{\_} 3$ \\
Spectral reflectance, OSM & $R_{-} 4$ \\
Spectral reflectance, Indices, GUF, OSM & $R_{-} 5$ \\
\hline
\end{tabular}

\section{DISCUSSION}

Based on the results in Section 3, feature importance and the choice of features for large-scale LCZ mapping will be discussed in this Section.

By comparing the blue, green, orange and aubergine bars in Fig. 3, we can see the different contributions of index measures, MPs, GUF and OSM, respectively. This contribution difference for ResNet is GUF>OSM > indices $>M P s$. Besides, Spectral reflectance, GUF is one of the feature configurations that provide the best accuracy (the other two are the baseline of Spectral reflectance only and the combination of Spectral reflectance, indices, GUF and OSM). It shows that GUF and OSM can improve the performance of the clas-

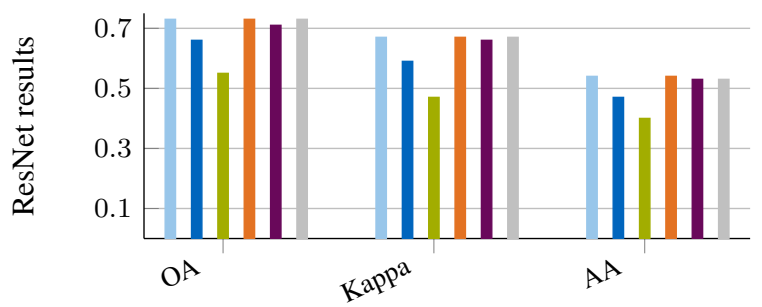

Fig. 3: OA, Kappa and AA resulting from different features. The values are averaged over the cross validation results. For each measure, from left to right, the bars are corresponding to the feature configurations $R_{-} 0, R_{-} 1, R_{-} 2, R_{-} 3, R_{-} 4$, and $R_{-} 5$ in Table. 1, respectively.

sifier, regarding generalization ability since the experiments are carried out in a cross validation manner. The highly possible reason is that GUF provides better distinguishability of urban LCZs from the background classes (water and vegetation), and the same holds for OSM (buildings) layer. In addition, MPs contribute the least, compared to indices, GUF and OSM, and the combination of Spectral reflectance and MPS provides the worst results for all configurations. This may be resulted from the limited spatial resolution of Sentinel-2 imagery. Another possibility is that ResNet already incorporates spatial information implicitly, via the convolutional filters.

The results of this study also provide a hint to the feature configuration choice for large-scale LCZ mapping. The best accuracy for the study case comes from Spectral reflectance or the combination of Spectral reflectance and GUF, or the combination of Spectral reflectance, Indices, GUF and OSM, among which no big difference exist. This possibly benefits from the deep features learned by the ResNet during training, thus making the additional input features not necessary. Therefore, the suggested input feature configuration is Spectral reflectance only, for large-scale or global urban LCZ 


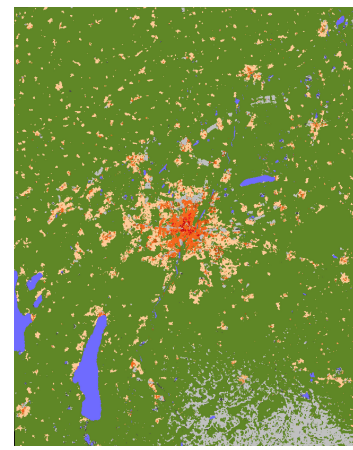

(a) LCZ map from $R_{-} O$

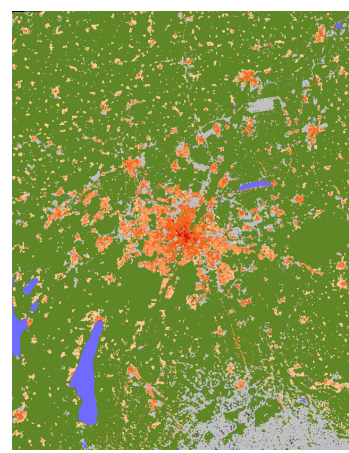

(b) LCZ map from $R \_1$

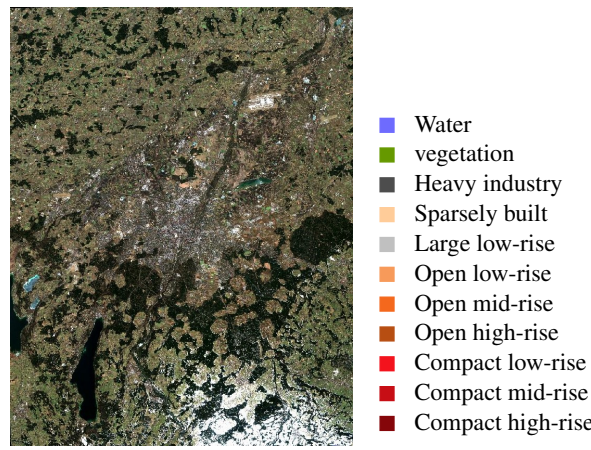

(c) Sentinel-2 RGB imagery

Fig. 4: The LCZ map of Munich produced using the feature configuration $R_{-} 0$ (a) and $R_{-} l$ (b), respectively, as well as the corresponding Sentinel-2 RGB imagery (c).

mapping with Sentinel-2 imagery using ResNet as the classifier, especially in areas where OSM are not available or areas that have seen significant development since the available GUF was produced.

While promising, Fig. 4 also shows clear difference in the produced maps from different configurations, while the ground truth can only be one or neither of them. The same holds for the produced maps in the literature [4].This indicates that additional efforts regarding complementary datasets, more advanced network, and LCZ map quality assessment are needed in the future.

\section{CONCLUSION AND OUTLOOK}

This paper presents an investigation of the applicability and importance of the features for urban LCZ classification, focusing on the globally available Sentinel-2 imagery. Investigated features include spectral reflectance, index measures, spatial features extracted by MPs and the external auxiliary data (GUF and OSM layers). Using ResNet, comparative experimental analysis was carried out in a large-scale study area located in Europe. Results based on cross validation show that GUF and OSM can contribute to the classification performance, while indices and MPs do not offer big benefits for ResNet. In addition, Spectral reflectance of Sentinel-2 only is suggested to be the input feature for large-scale urban LCZ mapping.

\section{ACKNOWLEDGEMENTS}

This work is jointly supported by the China Scholarship Council, the Helmholtz Association under the framework of the Young Investigators Group SiPEO (VH-NG-1018, www.sipeo.bgu.tum.de), and the European Research Council (ERC) under the European Union's Horizon 2020 research and innovation programme (grant agreement No. ERC-2016StG-714087, Acronym: So2Sat).

\section{REFERENCES}

[1] I. D. Stewart and T. R. Oke, "Local climate zones for urban temperature studies," Bull. Am. Meteorol. Soc., vol. 93, no. 12, pp. 1879-1900, 2012.

[2] Hung Chak Ho, Kevin Ka-Lun Lau, Ruby Yu, Dan Wang, Jean Woo, Timothy Chi Yui Kwok, and Edward Ng, "Spatial variability of geriatric depression risk in a high-density city: a data-driven socioenvironmental vulnerability mapping approach," International journal of environmental research and public health, vol. 14, no. 9, pp. 994, 2017.

[3] N. Yokoya, P. Ghamisi, and J. Xia, "Multimodal, multitemporal, and multisource global data fusion for local climate zones classification based on ensemble learning," Proc. IGARSS, 2017.

[4] N. Yokoya, P. Ghamisi, J. Xia, S. Sukhanov, R. Heremans, I. Tankoyeu, B. Bechtel, B. Le Saux, G. Moser, and D. Tuia, "Open data for global multimodal land use classification: Outcome of the 2017 ieee grss data fusion contest," IEEE J-STARS, 2018, DOI: 10.1109/JSTARS.2018.2799698.

[5] B. Bechtel, P. J. Alexander, J. Böhner, J. Ching, O. Conrad, J. Feddema, G. Mills, L. See, and I. Stewart, "Mapping local climate zones for a worldwide database of the form and function of cities," ISPRS Int. J. Geoinf., vol. 4, no. 1, pp. 199-219, 2015.

[6] M. Klotz, T. Kemper, C. Geiß, T. Esch, and H. Taubenböck, "How good is the map? a multi-scale cross-comparison framework for global settlement layers: Evidence from central europe," Remote Sens. Environ., vol. 178, pp. 191-212, 2016.

[7] J. Radoux, G. Chomé, D. C. Jacques, F. Waldner, N. Bellemans, N. Matton, C. Lamarche, R. D'Andrimont, and P. Defourny, "Sentinel2's potential for sub-pixel landscape feature detection," Int. J. Remote Sens., vol. 8, no. 6, 2016.

[8] X. Zhu and et al., "So2sat LCZ42 dataset," to appear.

[9] C.P. Qiu, M. Schmitt, P. Ghamisi, and X. Zhu, "Effect of the training set configuration on sentinel-2-based urban local climate zone classification," in IAPRS, 2018, in press.

[10] K. He, X. Zhang, S. Ren, and J. Sun, "Deep residual learning for image recognition," in Proc IEEE Comput Soc Conf Comput Vis Pattern Recognit, 2016, pp. 770-778.

[11] C. J. Tucker, "Red and photographic infrared linear combinations for monitoring vegetation," Remote Sens. Environ., vol. 8, no. 2, pp. 127$150,1979$.

[12] P. Ghamisi, M. Dalla Mura, and J. A. Benediktsson, "A survey on spectral-spatial classification techniques based on attribute profiles," IEEE Trans. Geosci. Remote Sens., vol. 53, no. 5, pp. 2335-2353, 2015. 